\title{
Two-stage model of visual backward masking: Sensory transmission and accrual of effective information as a function of target intensity and similarity
}

\author{
J. GERARD MUISE, RENAUD S. LEBLANC, MARC E. LAVOIE, and A. SERGE ARSENAULT \\ University of Moncton, Moncton, New Brunswick, Canada
}

\begin{abstract}
A functional model is used to describe the effect of target intensity and target-set similarity on backward visual masking. The model consists of two distinct stages of visual information processing. The first stage is related to sensory transduction and transmission and is assumed to require a finite and measurable amount of time during which performance remains at chance. The second stage, associated with central processing, is characterized by a negatively accelerated growth function reflecting the accrual of effective information. Results show that the duration of the transmission stage is inversely related to target intensity. Surprisingly, the rate of information accrual is an interactive function of both target intensity and target-set similarity. The pattern of results is consistent with the interpretation that both intensity and similarity mediate their effect through a common mechanism-the accrual of effective information.
\end{abstract}

The consensual view of visual backward masking holds that the perception of a target can be perturbed either at the peripheral level or at a more central level of processing. The distinction between peripheral and central visual processing stems mainly from Turvey's (1973) finding that two different classes of phenomena are observed in studies of visual backward masking. When masking depends on the energy in the target stimulus and is observed only with monoptic presentation, it is said to occur at a peripheral level of processing. However, when masking depends on the onset asynchronies of the target and the mask and is observed with dichoptic presentation, it is said to occur at a central level of processing. While peripheral masking can be characterized by a multiplicative rule relating target energy and minimum interstimulus interval (ISI; i.e., the elapsed time between stimulus offset and mask onset) required for asymptotic performance, central masking, on the other hand, can be characterized by an additive rule relating target duration and minimum ISI.

A number of researchers (Alpern, 1968; Lupker \& Massaro, 1979; Rumelhart, 1970; Turvey, 1973) have examined how performance varies as a function of processing time. They have generally reported that the early part of this function corresponds to either an above chance but

\footnotetext{
We thank Roger Boudreau for technical assistance in building the experimental apparatus. Preliminary work leading to this paper was made possible by a grant from the Centre d'études sur le vieillissement, Université de Moncton. Request for reprints should be addressed to either of the first two authors at the following address: Laboratoire de psychologie cognitive, Département de psychologie, Université de Moncton, Moncton, NB E1A 3E9, Canada.
}

decreasing level of performance or a relatively stable level of chance responding as ISI increases. The specific shape of this early segment depends on the energic and the featural relationships between the target and the mask. In the later part of the function, a rapid increase to an asymptotic level is reported. In auditory masking, Massaro $(1970,1972,1974)$ has shown that an exponential growth function describes quite adequately the data obtained for the later portion of the masking curve. The model that he used has the following form:

$$
d^{\prime}=\alpha\left[1-e^{-\theta t}\right],
$$

where $\alpha$ represents the amount of information available in the stimulus configuration, $\theta$ represents the rate of information extraction, $e$ is the base of the natural logarithm, and $t$ represents the stimulus onset asynchrony (SOA; i.e., the elapsed time between stimulus onset and mask onset). Lupker and Massaro (1979) have presented an extended version of the model, which they applied to visual information processing. In this version, the parameters can vary as a result of the attentional and task demands brought to bear in the perceptual processing stages.

In all these models, it is assumed that the $x$-intercept of the growth function is at time zero-that is, at targetstimulus onset. It is probable, however, that there is a finite amount of time associated with what may be called sensory transduction and transmission. This notion has been alluded to in studies of the Pulfrich effect (Alpern, 1968; Lit, 1949), of visual backward masking (Rumelhart, 1970), and of reaction time (Nissen, 1977). Through selection of the appropriate relationship between the target and mask, it may be possible to completely interfere with the peripheral processing of the target, either by in- 
terrupting it (Averbach \& Coriell, 1961; Sperling, 1963) or by submerging the target information in a deluge of noise (Eriksen, 1966). Complete interference during sensory transmission would be reflected in a period of chance performance that could extend to tens of milliseconds or more. On the other hand, if the interference occurs beyond the time required for transduction and transmission, performance should increase above chance according to an exponential growth function.

More specifically, we propose that for any given target-mask configuration that produces full masking at a very short ISI, there will follow a period of chance performance. The extent of this period depends on the time that it takes for the target information to traverse the peripheral network and on the relative speed of transmission of the target and the mask. Estimates of this transmission time can be obtained by determining the pivotal ISI that allows performance to rise just above chance level for the specific target-mask configuration that is being used. We refer to this interval as transmission lag $\left(T_{\text {lag }}\right)$.

Following this initial period of chance performance, we assume a period of central information processing. We view this processing as an information accrual process that feeds into a decision network. If the accrual process is interrupted, or disrupted, before it has reached completion, the decision network provides an identification on the basis of the partial information already processed.

In its simplest expression, the model that we propose has the following form:

$$
d^{\prime}=\alpha\left[1-e^{-\theta\left(\mathrm{ISI}-T_{\mathrm{lag}}\right)}\right]
$$

for ISI $\geq T_{\text {lag }}$, and

$$
d^{\prime}=0,
$$

for ISI $<T_{\text {lag }}$, where $\alpha$ represents the amount of information available in the stimulus configuration, $\theta$ represents the rate of information accrual, $e$ is the base of the natural logarithm, ISI represents the duration of the interstimulus interval in milliseconds, and $T_{\text {lag }}$ corresponds to the $x$-intercept of the growth function in milliseconds.

This formulation is reminiscent of the speed-accuracy tradeoff memory retrieval function studied extensively by Wickelgren and his colleagues (Corbett, 1977; Corbett \& Wickelgren, 1978; Dosher, 1976; Wickelgren \& Corbett, 1977). They have shown "that the time course of retrieval is well approximated by an initial period of chance accuracy $\left(d^{\prime}=0\right)$ up to time $T=\delta$ (the intercept) followed by an exponential approach to asymptotic accuracy" (Corbett \& Wickelgren, 1978, p. 3).

Our aim in the present study is to describe the functional relationship between performance and duration of the ISI in visual backward masking. In an attempt to understand the dynamics of visual information processing, two other variables, target stimulus intensity and target set similarity, were manipulated.

It is a well-documented fact that increases in stimulus intensity increase the speed of sensory transduction and transmission. This is confirmed by the existence of an in- verse relationship between stimulus intensity and the response latency of retinal ganglion cells (Cleland \& Enroth-Cugell, 1970; Hartline, 1938; Levick, 1973), between stimulus intensity and the latency of the evoked response (Vaughan, Costa, \& Gilden, 1966; Vaughan \& Hull, 1965), and between stimulus intensity and reaction time (Cattell, 1886; Mansfield, 1973; Minucci \& Connors, 1964; Piéron, 1914, 1920; Vaughan et al., 1966). Therefore, we predict that $T_{\text {lag }}$ will be an inverse function of target stimulus intensity - that is, that an increase in target intensity will shorten $T_{\text {lag }}$.

The similarity relationships between the target and the mask (i.e., pattern mask versus random-noise mask), between the target and the distractors in multiitem displays (Gardner, 1973; Kleiss \& Lane, 1986; Lupker \& Massaro, 1979), and between two members of a stimulus pair (Krueger, 1987) have produced strong effects in visual masking. However, to our knowledge, the effect of variations in similarity relationships within the set of target items has never been studied explicitly in a single-letter presentation paradigm. In fact, many authors have avoided such parametric manipulations, preferring instead to use letters that are "approximately equally recognizable and equally confusable, one with another" (Eriksen \& Collins, 1965, p. 345). If all the items in the target set are highly similar, decisions will have to be made on the basis of a more compact distance-density space (Appelman \& Mayzner, 1982; Krumhansl, 1978). A target set in which the items are quite different from each other will result in a sparse distance-density space. Increasing the within-target-set similarity, either by increasing the number of common features or by decreasing the distinctive information (Tversky, 1977), should render the operation of the decision process more difficult. This increase in difficulty should be reflected in a decrease in the effective rate of processing, or, in terms of the model, a decrease in the $\theta$ parameter. Changes in the target-set similarity should have no effect on the time required for peripheral processing, or $T_{\text {lag }}$.

Therefore, the intensity manipulation should affect $T_{\text {lag }}$ while the similarity manipulation should affect $\theta$.

\section{METHOD}

\section{Observers}

Eight undergraduate males with corrected-to-normal vision served as volunteers. Their ages varied from 20 to 30 years with a mean age of 23 .

\section{Material}

The stimuli were presented on a Leader LB0-508A oscilloscope with a greenish-tint fast-decay P31 phosphor (decays to $1 \%$ intensity at $.25 \mathrm{msec}$ after display onset). Stimulus presentation was controlled by means of an Apple II + microcomputer with a 1-MHz direct-memory-access circuit, a multiplexer, and a digital-to-analog (D/A) conversion circuit. The last three circuits were designed and constructed at the Laboratoire de psychologie cognitive. The $x$ and $y$ voltages were generated by two Datel DACHF8BMC D/A 8-bit converters with a settling time of $15 \mathrm{nsec}$. The intensity or $z$ voltage was generated by a Datel DACHI10B D/A 10-bit converter 
with a settling time of $25 \mathrm{nsec}$. A $100-\mathrm{MHz}$ bandwidth operational amplifier circuit (Datel AM-450-2) allowed the voltage values to vary between 0.8 and $3.2 \mathrm{~V}$. Full blanking was obtained when a digital value of 0 was sent to the D/A circuit. This setup allowed the transmission of one dot (three coordinates: horizontal displacement, vertical displacement, and intensity) on the CRT screen within $3 \mu \mathrm{sec}$. All testing was conducted in a Ray Proof shielded soundproof room.

The observers looked through a visor placed at a distance of $1 \mathrm{~m}$ from the CRT screen. The central part of the screen was surrounded by a circular piece of matte black cardboard that limited the field of vision to a circular area with a radius of $4 \mathrm{~cm}$.

The letters "CGOQ" constituted the high-similarity stimulus set, and the letters "IOSX" constituted the low-similarity stimulus set. Except for the letter "I," which was narrower, the target stimuli were $1.0 \mathrm{~cm}$ wide and $1.6 \mathrm{~cm}$ high, subtending visual angles of $0.57^{\circ}$ and $0.92^{\circ}$. The letters consisted of between 189 and 386 dots and had a definite stroke-like appearance. The time required to write each letter once on the screen varied between 0.554 and $1.131 \mathrm{msec}$. A grid pattern made up of diagonal, horizontal, and vertical line segments served as the masking stimulus. The mask was $3.2 \mathrm{~cm}$ wide and $2.6 \mathrm{~cm}$ high, subtending visual angles of $1.83^{\circ}$ and $1.50^{\circ}$. Reproductions of the target stimuli and the mask are presented in Figure 1.

In the case of the letters, the intensity of each dot was controlled by a 700 -nsec pulse of the $z$-axis, which enabled the voltage produced by one of the following digital values: $150,200,250$, or 300 . These digital values corresponded to calibration intensities of $0.57,0.70$, 0.86 , and $1.06 \mathrm{~cd} / \mathrm{m}^{2}$. The dots composing the mask were produced with a digital value of 700 , corresponding to a calibration intensity of $5.48 \mathrm{~cd} / \mathrm{m}^{2}$. The calibration procedure measured the luminance of a $26 \times 26$ square matrix corresponding to an area of $4 \mathrm{~cm}^{2}$ with a Pentax Spotmeter $V$, while the display was refreshed every $7.8 \mathrm{msec}$.

\section{Procedure}

Each trial started with the presentation of an auditory signal, followed by a dim fixation point at the center of the circular area of the screen. The observer indicated that he was ready for the presentation of the target by pressing on a footpedal. The fixation point would then disappear, and after a delay of $150 \mathrm{msec}$, the target was presented at the center of the circular viewing area. Following the ISI, the mask was presented for 100 cycles, each cycle requiring approximately $3 \mathrm{msec}$. Being wider and higher than the individual target stimuli, the mask extended beyond the area covered by them.

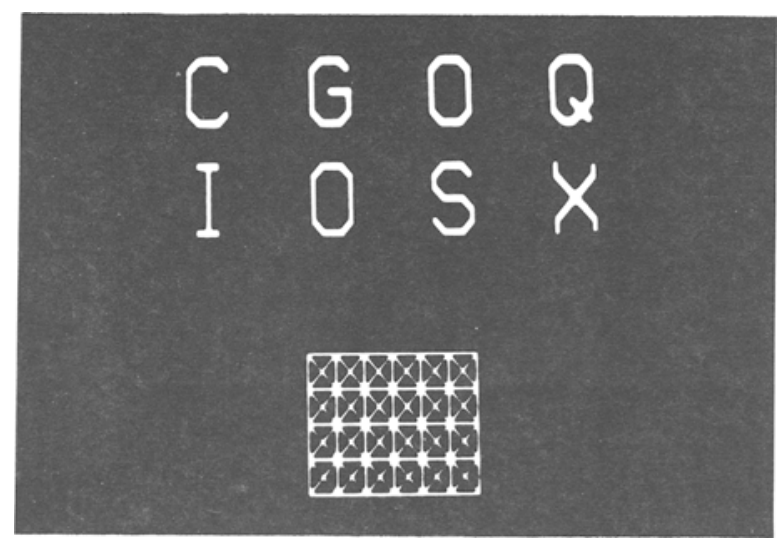

Figure 1. Reproductions of the high-similarity stimulus set, the low-similarity stimulus set, and the mask.
Sixteen different ISI values were used: $5,10,15,20,25,30,40$, $50,60,70,80,90,100,120,160$, and $200 \mathrm{msec}$.

Following the mask, the four possible target stimuli were presented at the bottom of the circular area in a horizontal array. The array was $4.2 \mathrm{~cm}\left(2.40^{\circ}\right)$ wide and $0.9 \mathrm{~cm}\left(0.52^{\circ}\right)$ high. The observer indicated his response by moving a cursor above the stimulus that he believed had been presented and pressing a button. A correct response was indicated by an auditory signal that differed in frequency from the tone used as a ready signal. No tone was presented in the case of an incorrect response.

Each subject participated in two experimental sessions. Within each session, either the high-similarity stimulus set or the lowsimilarity stimulus set was used. The order of presentation of the two stimulus sets was counterbalanced across subjects. A session consisted of three blocks of 256 trials each (all combinations of 4 stimuli, 4 intensity levels, and 16 ISI values). The order of presentation of the trials within each block was randomly determined, and it differed across blocks and observers.

At the beginning of each session, the observers were presented with the instructions and 14 practice trials with descending ISI values. This period allowed the subjects to become dark-adaptated to the very low ambient lighting.

\section{RESULTS}

For each subject, the average proportion of correct responses, based on 12 observations ( 4 stimuli and 3 blocks), was calculated for the 16 ISI values at each of the 8 experimental conditions ( 2 levels of stimulus similarity $\times 4$ levels of stimulus intensity). These average proportions were then transformed to four-alternative $d^{\prime}$ values, using Algorithm 2 from Smith (1982).

A $2 \times 4$ ( 2 levels of similarity and 4 levels of intensity) repeated measures analysis of variance, applied to the $d^{\prime}$ values, revealed reliable main effects of similarity $[F(1,7)=8.68, p<.05]$, intensity $[F(3,21)=111.34$, $p<.001]$, and ISI $[F(15,105)=335.82, p<.001]$. Performance increased as similarity decreased, as intensity increased, and as ISI increased. Of particular interest for the hypotheses under consideration, the following three interactions were significant: similarity $\times \operatorname{ISI}[F(15,105)$ $=2.16, p<.05]$, intensity $\times \operatorname{ISI}[F(45,315)=13.11$, $p<.001]$, and similarity $\times$ intensity $\times$ ISI $[F(45,315)$ $=1.92, p<.001]$. Visual inspection of the data points presented in Figures 2 and 3 indicates that the $x$-intercept occurs at shorter ISI values as intensity increases. The level of performance increases more rapidly at higher intensities. In addition, the effect of intensity on the rate of increase in the level of performance appears to be stronger in the low-similarity condition than in the highsimilarity condition.

The changes that occur as a result of the two manipulations appear quite systematic and might be captured by a version of the model that takes into account the effect of intensity on $T_{\text {lag }}$ and the joint effect of intensity and similarity on $\theta$. We tested a version of the model in which linear weights were assigned to intensity and similarity. We defined $\theta_{\text {is }}$ as a joint multiplicative function of intensity and similarity:

$$
\theta_{\mathrm{is}}=\theta_{\mathrm{i}} \theta_{\mathrm{s}},
$$


High Similarity
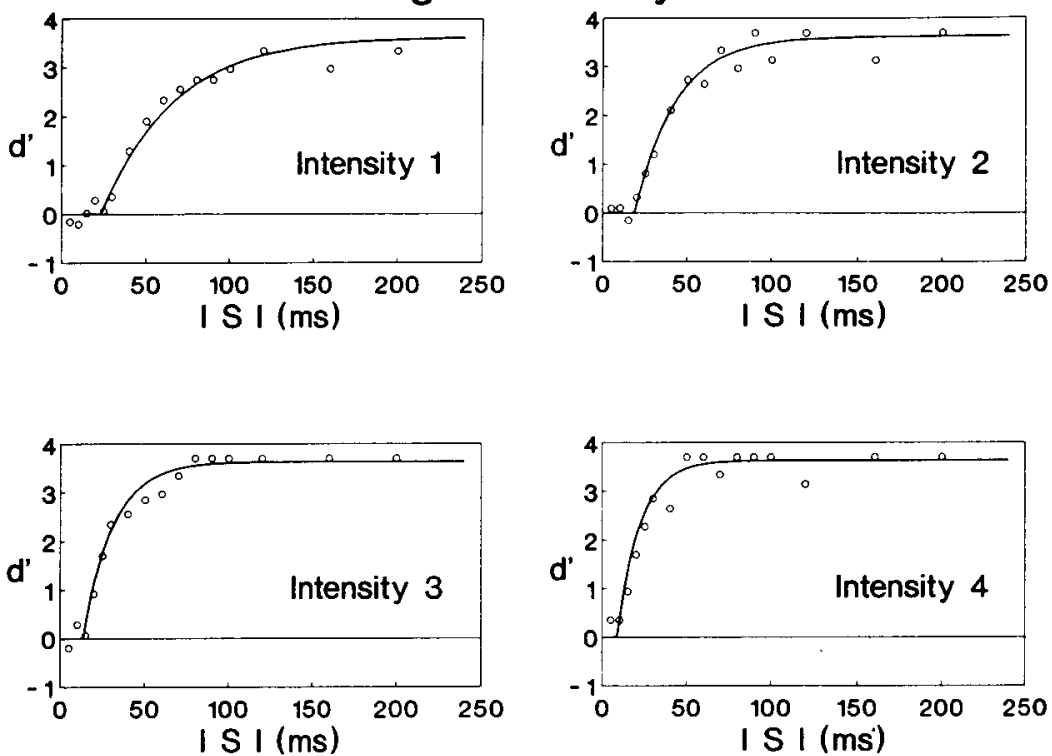

Figure 2. Predicted and observed $d^{\prime}$ values for the interactive model as a function of intensity (high-similarity stimulus set).

Low Similarity
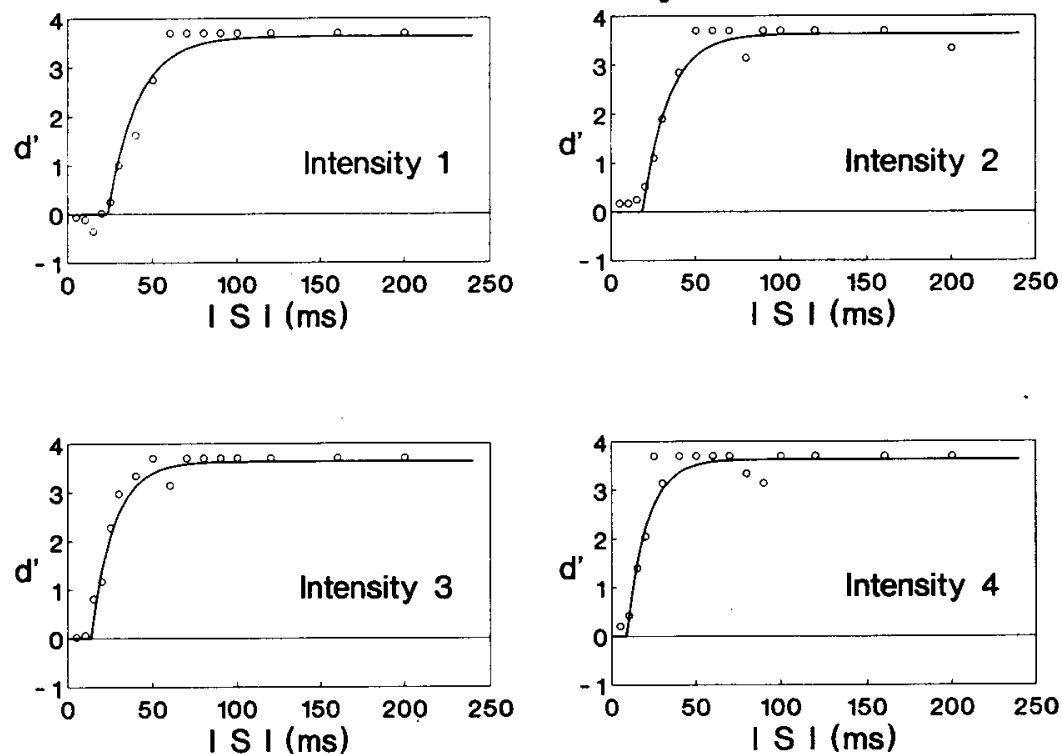

Figure 3. Predicted and observed $d^{\prime}$ values for the interactive model as a function of intensity (low-similarity stimulus set). 
where $\theta_{\text {is }}$ is the rate of information accrual for a given intensity and similarity condition, $\theta_{\mathrm{i}}$ is the contribution of a given intensity on the accrual rate, and $\theta_{\mathrm{s}}$ is the contribution of a given similarity level on the accrual rate.

We further defined the effect of intensity on $T_{\text {lag }}$ as:

$$
T_{\text {lag i }}=a_{\mathrm{T}}+b_{\mathrm{T}} \text { intensity; }
$$

the effect of intensity on $\theta$ as:

$$
\theta_{\mathrm{i}}=a_{\mathrm{I}}+b_{\mathrm{I}} \text { intensity; }
$$

and the effect of similarity on $\theta$ as:

$$
\theta_{\mathrm{s}}=a_{\mathrm{s}}+b_{\mathrm{s}} \text { similarity, }
$$

where $a_{\mathrm{T}}, a_{\mathrm{I}}$, and $a_{\mathrm{S}}$ represent the intercept of the linear functions relating, respectively, intensity to $T_{\text {lag }}$, intensity to $\theta_{\mathrm{i}}$, and similarity to $\theta_{\mathrm{s}}$; and where $b_{\mathrm{T}}, b_{\mathrm{I}}$, and $b_{\mathrm{S}}$ represent the slopes of the corresponding linear functions. Although three linear functions were used to relate intensity and similarity to $T_{\text {lag }}$ and $\theta$, extrapolating the linearity to other intensity and similarity ranges may be inappropriate without further empirical investigation.

The complete model can be expressed as follows:

$$
d^{\prime}=\alpha\left[1-e^{-\theta_{\text {is }}\left(\text { ISI }-T_{\text {lasi }}\right)}\right],
$$

for ISI $\geq T_{\text {lagi }}$, and

$$
d^{\prime}=0,
$$

for ISI $<T_{\text {lag } \mathrm{i}}$

This model was applied to the $d^{\prime}$ transformation of the proportions of correct responses averaged across subjects. Arbitrary scale values of $1,2,3$, and 4 were used to represent increasing intensity values, since the logarithms of the intensities used were linear. An arbitrary scale value of 1 was used to represent the high-similarity condition, and a value of 2 was used to represent the low-similarity condition. The parameter estimates were obtained with STEPIT (Chandler, 1969) and are presented in Table 1.

With each increasing level of stimulus intensity, $5 \mathrm{msec}$ were subtracted from the intercept value of $28 \mathrm{msec}$ (i.e., the least intense stimulus condition yielded a $T_{\text {lag }}$ of $23 \mathrm{msec}$; the most intense stimulus condition yielded a $T_{\text {lag }}$ of $\left.8 \mathrm{msec}\right)$. The parameters related to the rate of ef-

Table 1

Parameter Estimates for the Interactive Model

\begin{tabular}{cc}
\hline \multicolumn{1}{c}{ Parameter } & $\begin{array}{c}\text { Interactive } \\
\text { Model }\end{array}$ \\
\hline$T_{\text {lag }}$ (in milliseconds) & 28 \\
Intercept & -5 \\
Slope & \\
$\theta_{\mathrm{i}}$ & 14.46 \\
Intercept & 9.88 \\
Slope & \\
$\theta_{3}$ & 0.07 \\
Intercept & 1.10 \\
Slope & 3.64 \\
\hline
\end{tabular}

Table 2

Predicted $T_{\text {las }}$ Values as a Function of Intensity and $\theta_{\text {is }}$ Values as a Function of Intensity and Similarity

\begin{tabular}{lcccc}
\hline & \multicolumn{4}{c}{ Intensity } \\
\cline { 2 - 5 } & 1 & 2 & 3 & 4 \\
\hline$T_{\text {las }}$ (in milliseconds) & 23 & 18 & 13 & 8 \\
$\theta_{\text {is }}$ & & & & \\
Low similarity & 55.25 & 77.68 & 100.11 & 122.53 \\
High similarity & 28.48 & 40.04 & 51.60 & 63.16 \\
\hline
\end{tabular}

fective information accrual are also presented in Table 1. The predicted $T_{\text {lag }}$ and $\theta_{\text {is }}$ values are presented in Table 2 . The effect of increasing the similarity in the present study was to approximately halve the processing rate. Similarly, the processing rate approximately doubled over the range of intensities used.

Figures 2 and 3 present the observed and predicted $d^{\prime}$ values for the interactive model (for the high-similarity and low-similarity conditions, respectively). The correspondence of the interactive model to the data is substantial. The root mean square of the deviations between the observed and the predicted $d^{\prime}$ values was 0.231 . However, the parameter estimates are derived from averaged data, which provide no estimate of variability. Consequently, the interactive model was applied to each subject's data, providing estimates of $T_{\text {lag }}$ and of the joint $\theta_{\text {is }}$ values predicted for each intensity and similarity condition. A repeated measures analysis of variance on $T_{\text {lag }}$ revealed a significant inverse effect of intensity $[F(3,21)$ $=135.25, p<.001]$. A $2 \times 4$ repeated measures analysis of variance on the $\theta_{\text {is }}$ values revealed significant main effects of intensity $[F(3,21)=14.51, p<.001]$ and similarity $[F(1,7)=15.45, p<.01]$, as well as a significant intensity $\times$ similarity interaction $[F(3,21)=$ $11.35, p<.001]$.

These analyses demonstrate the reliability of the effects on the parameters of the model and confirm the pattern of results obtained with the averaged data.

\section{GENERAL DISCUSSION}

\section{Two-Stage Model}

We propose a two-stage model of visual backward masking. The first stage is operationally defined as the initial period of chance performance. The second stage is characterized by a negatively accelerated exponential growth in performance based on the process of accrual of effective information. By effective information, we refer to any information that is useful in distinguishing among the elements of the target ensemble.

\section{Travel Time}

We have referred generically to $T_{\text {lag }}$ as sensory transmission time. By using this term, we do not aim to espouse any unique theoretical view. Rather, we want to denote something similar to what Nissen (1977) referred to as "travel time" when she discussed the effect of in- 
tensity on the latency of the evoked potential. The finding of an inverse relationship between stimulus intensity and the latency of the evoked response contradicts most early models of simple reaction time (Grice, 1968; Luce \& Green, 1972; McGill, 1963). In those models, it was proposed that intensity manipulations have their effect on the rate of firing of neural units and not on the amount of time required for the information to reach the central locus of processing. However, Vaughan et al. (1966) found that stimulus intensity produced identical effects on simple reaction time and on the latency of the evoked response. Also, at the level of the retinal ganglion cells, a number of researchers (Cleland \& Enroth-Cugell, 1970; Hartline, 1938; Levick, 1973) have reported a 10-msec decrease in the latency of the initial pulse for each log unit change in intensity.

In attempting to delineate the locus of intensity effects, Posner (1978) stated that "intensity manipulations may change the response criterion, the time at which information starts to accrue (i.e., leave the $x$-axis), the rate at which information grows, or any combination of these" (pp. 112-113). The results of the present study clearly favor one particular combination; that is, intensity manipulations change both the start of information accrual and the rate of growth.

$T_{\text {lag }}$, or the start of information accrual, is only an estimate of absolute travel time, since it reflects the difference in arrival time of the target and mask information at the locus of central processing. Since increases in intensity apparently increase the speed of transmission, information from a bright mask should be transmitted faster than information from a dim target. In this case, $T_{\text {lag }}$ would be an underestimate of the actual target travel time. Hence $T_{\text {lag }}$ should be an orderly function of the relative intensity of the target and the mask.

\section{Effective Information Accrual}

The origin of the accrual function is at $T_{\text {lag }}$, where performance is still at chance. Any departure from chance performance represents the accumulation of effective information-that is, information that is relevant in determining which target item was presented. Effective information accrual is not synonymous with information processing and/or feature extraction. Although feature extraction may operate at a constant rate across many stimulus configurations and tasks, in any given situation only a portion of this information is effective or distinctive.

For example, although features may be extracted from the target letter " $O$ " at a constant rate, independently of whether it has to be distinguished from similar items ("CGQ") or from dissimilar items ("ISX"), the rate of processing of effective or distinctive information differs substantially. The extraction of any information pertaining to the circular aspects of the stimulus configuration should be immediately pertinent with the dissimilar set, whereas it should be much less informative with the similar set. The measurement of effective information may be related to a number of conceptual frameworks, such as the proximity notions of Shepard (1962a, 1962b, 1974), the set-theoretic notions of Tversky (1977), the distance-density notions of Krumhansl (1978), the fuzzy propositional notions of Oden (1979), or the distinctiveness notions of Donderi (1988).

Although intensity did affect $T_{\text {lag }}$ independently of similarity, it interacted strongly with similarity at the central or effective information accrual stage. When we designed the experiment, we did not expect intensity and similarity to interact so strongly. Indeed, the traditional models of information processing would probably predict that intensity would operate on the rate of feature extraction, while similarity would operate on a subsequent stage characterized by decisional processes (Gardner, 1973).

For the second stage, we suggest a more parsimonious one-process model, in which effective information builds up over time via the accrual of distinctive information. We consider that both intensity and similarity manipulations result in changes in the distinctiveness of the items within the target ensemble. One possible explanation may be formulated in terms of linear systems analysis. Decreases in target intensity decrease the spectral energy in all bands proportionately. Since the dominant energy is usually in the low frequencies, high spatial frequency information is the first to be lost or to become unavailable. By reducing the amount of high-frequency information, either the separation of the items in the multidimensional space becomes less with a corresponding increase in the density of the region, or the proportion of shared common features increases. Furthermore, this differential filtering effect is compounded by the fact that the physiological filtering mechanism of the transient neurons (Breitmeyer \& Ganz, 1976) has already attenuated or ignored the spectral energy of the higher frequencies.

Thus, a reduction in intensity and/or an increase in similarity may reduce the effective rate of information accrual. Similarity operates by rendering more of the extracted information irrelevant for the task at hand, whereas intensity operates by filtering out the more distinctive information. Such a common effect of intensity and similarity was also considered by Pachella (1974), who discussed the possibility of "varying discriminability by reducing stimulus contrast" and of "changing discriminability by increasing stimulus similarity" (p. 65).

Although we have favored the interpretation that the factor that determines above-chance performance on this task is the rate of accrual of effective information, it is quite possible that the observed interaction between intensity and similarity is the result of changes in two independent processes. Using reaction time data, Grice (1968) has aptly shown that it is possible for two manipulations that supposedly affect two independent stages to have an interactive effect on performance. For example, the basic rate of information accrual could be a function of any manipulation that modifies the quality of the information reaching the central processor, such as intensity. Hence, a bright target would be processed at a faster rate, centrally, than a dim target would. In addition, an 
increase in stimulus similarity could require the use of a stricter criterion by the subsequent decision process.

Because manipulations of intensity and similarity interact multiplicatively at the information accrual stage, they might appear to be equivalent manipulations that result in identical signal-to-noise ratios. Isometric performance functions could be obtained through manipulation of either of the two variables. However, in terms of information processing, a change in intensity is not equivalent to a change in similarity. Whereas zero intensity corresponds to true zero information, zero distinctiveness (or identity) corresponds to effective zero information. In the case of zero intensity, no information is available for processing, whereas in the case of identity, a substantial amount of information may be processed, but it would not be useful in distinguishing between two identical items.

In summary, we have presented a two-stage model of visual backward masking. The initial stage is associated with sensory transduction and transmission, and it is characterized by an initial period of chance responding. The second stage corresponds with the accrual of effective information, and it is characterized by a negatively accelerated exponential growth in performance. The duration of the sensory transmission stage is inversely related to target intensity. The rate of effective information accrual is an interactive function of both target intensity and target-set similarity.

\section{REFERENCES}

AlPern, M. (1968). A note on visual latency. Psychological Review, 75, 260-264.

Appelman, I. B., MAyzner, M. S. (1982). Application of geometric models to letter recognition: Distance and density. Journal of Experimental Psychology: General, 111, 60-100.

Averbach, E., \& Coriell, A. S. (1961). Short-term memory in vision. Bell Systems Technical Joumal, 40, 309-328.

BreITMEYER, B. G. , GANZ, L. (1976). Implications of sustained and transient channels for theories of visual pattern masking, saccadic suppression, and information processing. Psychological Review, 83, 1-36.

Catrell, J. M. (1886). The influence of intensity of the stimulus on the length of the reaction time. Brain, 8, 512-515.

ChandLer, J. P. (1969). STEPIT: Finds local minima of a smooth function of several parameters (CPA312). Behavioral Science, 14, 105-115.

Cleland, B. G., Enroth-Cugell, C. (1970). Quantitative aspects of gain and latency in the cat retina. Joumal of Physiology, 206, 73-91.

Corbetr, A. T. (1977). Retrieval dynamics for rote and visual image mnemonics. Journal of Verbal Learning \& Verbal Behavior, 16 , 234-246.

Corbett, A. T., Wickelgren, W. A. (1978). Semantic memory retrieval: Analysis by speed-accuracy tradeoff functions. Quarterly Journal of Experimental Psychology, 30, 1-15.

DONDERI, D. C. (1988). Information measurement of distinctiveness and similarity. Perception \& Psychophysics, 44, 576-584.

Dosher, B. A. (1976). The retrieval of sentences from memory: A speed-accuracy study. Cognitive Psychology, 8, 291-310.

ERIKSEN, C. W. (1966). Temporal luminance summation effects in backward and forward masking. Perception \& Psychophysics, 1, 87-92.

Eriksen, C. W., \& ColuINs, J. F. (1965). Reinterpretation of one form of backward and forward masking in visual perception. Joumal of Experimental Psychology, 70, 343-351.

GARDNER, G. T. (1973). Evidence for independent parallel channels in tachistoscopic perception. Cognitive Psychology, 4, 130-155.
GrICE, G. R. (1968). Stimulus intensity and response evocation. Psychological Review, 75, 359-373.

HARTLINE, H. K. (1938). The response of single optic nerve fibers of the vertebrate eye to illumination of the retina. American Jourmal of Physiology, 121, 400-415.

KLeISS, J. A., \&ANE, D. A. (1986). Locus and persistence of capacity limitations in visual information processing. Joumal of Experimental Psychology: Human Perception \& Performance, 12, 200-210.

KRUEGER, L. E. (1987). Effect of backward masking on same-different judgments. Perception \& Psychophysics, 41, 375-381.

KrumhansL, C. L. (1978). Concerning the applicability of geometric models to similarity data: The interrelationship between similarity and spatial density. Psychological Review, 85, 445-463

LEVICK, W. R. (1973). Variations in the response latency of cat retinal ganglion cells. Vision Research, 13, 837-853.

LIT, A. (1949). The magnitude of the Pulfrich stereophenomenon as a function of binocular differences of intensity at various levels of illumination. American Joumal of Psychology, 62, 159-181.

LUCE, R. D., \&REEN, D. M. (1972). A neural timing theory for response times and the psychophysics of intensity. Psychological Review, 79, 14-57.

LUPKER, S. J., \& MASSARo, D. W. (1979). Selective perception without confounding contributions of decision and memory. Perception \& Psychophysics, 25, 60-69.

MANSFIELD, R. J. W. (1973). Latency functions in human vision. $V i-$ sion Research, 13, 2219-2234.

MAssARo, D. W. (1970). Perceptual processes and forgetting in memory tasks. Psychological Review, 77, 557-567.

Massaro, D. W. (1972). Stimulus information vs processing time in auditory pattern recognition. Perception \& Psychophysics, 12, 50-56.

Massaro, D. W. (1974). Perceptual units in speech recognition. Journal of Experimental Psychology, 102, 199-208.

McGilL, W. J. (1963). Stochastic latency mechanisms. In R. D. Luce, R. R. Bush, \& E. Galanter (Eds.), Handbook of mathematical psychology (Vol. 1, pp. 309-360). New York: Wiley.

Minucci, P. K., \& Connors, M. M. (1964). Reaction time under three viewing conditions: Binocular, dominant eye, and nondominant eye. Journal of Experimental Psychology, 67, 268-275.

Nissen, M. J. (1977). Stimulus intensity and information processing. Perception \& Psychophysics, 22, 338-352.

ODEN, G. C. (1979). A fuzzy logical model of letter identification. Journal of Experimental Psychology: Human Perception \& Performance, 5, 336-352.

PACHella, R. G. (1974). The interpretation of reaction time in information-processing research. In B. H. Kantowitz (Ed.), Human information processing: Tutorials in performance and cognition (pp. 41-82). Hillsdale, NJ: Erlbaum.

Piéron, H. (1914). Recherches sur les lois de variation des temps de latence sensorielle en fonction des intensités excitatrices. Année Psychologique, 20, 17-96.

Piéron, H. (1920). Nouvelles recherches sur l'analyse du temps de latence sensorielle et la loi qui relie le temps à l'intensité d'excitation. Année Psychologique, 22, 58-142.

Posner, M. I. (1978). Chronometric explorations of mind. Hillsdale, NJ: Erlbaum.

Rumelhart, D. E. (1970). A multicomponent theory of the perception of briefly exposed visual displays. Journal of Mathematical Psychology, 7, 191-218.

SHEPARD, R. N. (1962a). The analysis of proximities: Multidimensional scaling with an unknown distance function: Part I. Psychometrika, 27, $125-140$.

SHEPARD, R. N. (1962b). The analysis of proximities: Multidimensional scaling with an unknown distance function: Part I. Psychometrika, 27, 219-246.

SHEPARD, R. N. (1974). Representation of structure in similarity data: Problems and prospects. Psychometrika, 39, 373-421.

SMITH, J. E. K. (1982). Simple algorithms for M-alternative forcedchoice calculations. Perception \& Psychophysics, 31, 95-96.

SPERLING, G. (1963). A model for visual memory tasks. Human Factors, $5,19-31$. 
TURveY, M. T. (1973). On peripheral and central processes in vision: Inferences from an information processing analysis of masking with patterned stimuli. Psychological Review, 80, 1-52.

TVERSKy, A. (1977). Features of similarity. Psychological Review, 84, 327-352.

Vaughan, H. G., Costa, L. D., * Gilden, L. (1966). The functional relation of visual evoked response and reaction time to stimulus intensity. Vision Research, 6, 645-656.

VAUghan, H. G., \& HUlL, R. C. (1965). Functional relation between stimulus intensity and photically evoked cerebral responses in man. Nature, 206, 720-722.

Wickelgren, W. A., \& CorbetT, A. T. (1977). Associative interference and retrieval dynamics in yes-no recall and recognition. Journal of Experimental Psychology: Human Learning \& Memory, 3, 189-202.

(Manuscript received November 6, 1990; revision accepted for publication March 15, 1991.) 